Pacific Journal of Mathematics

OPERATORS WITH FINITE ASCENT AND DESCENT 


\title{
OPERATORS WITH FINITE ASCENT AND DESCENT
}

\author{
S. R. CARADUS
}

Let $X$ be a Banach space and $T$ a closed linear operator with range and domain in $X$. Let $\alpha(T)$ and $\delta(T)$ denote, respectively, the lengths of the chains of null spaces $N\left(T^{K}\right)$ and ranges $R\left(T^{K}\right)$ of the iterates of $T$. The Riesz region $\Re_{T}$ of an operator $T$ is defined as the set of $\lambda$ such that $\alpha(T-\lambda)$ and $\delta(T-\lambda)$ are finite. The Fredholm region $\widetilde{\mho}_{T}$ is defined as the set of $\lambda$ such that $n(T-\lambda)$ and $d(T-\lambda)$ are finite, $n(T)$ denoting the dimension of $N(T)$ and $d(T)$ the codimension of $R(T)$. It is shown that $\widetilde{\mho}_{T} \cap \widetilde{\Im}_{T}$ is an open set on the components of which $\alpha(T-\lambda)$ and $\delta(T-\lambda)$ are equal, when $T$ is densely defined, with common value constant except at isolated points. Moreover, under certain other conditions, $\Re_{T}$ is shown to be open. Finally, some information about the nature of these conditions is obtained.

Let $X$ denote an arbitrary Banach space and suppose that $T$ is a linear operator with domain $D(T)$ and range $R(T)$ in $X$. We shall write $N(T)$ for the nullspace, $N(T)=\{x \in D(T)$ : $\quad T x=0\}$.

Let $D\left(T^{n}\right)=\left\{x: \quad x, T x, \cdots, T^{n-1} x \in D(T)\right\}$ and define $T^{n}$ on this domain by the equation $T^{n} x=T\left(T^{n-1} x\right)$ where $n$ is any positive integer and $T^{\circ}=I$. It is a simple matter to verify that $\left\{N\left(T^{k}\right)\right\}$ forms an ascending sequence of subspaces. Suppose that for some $k, N\left(T^{k}\right)=$ $N\left(T^{k+1}\right)$; we shall then write $\alpha(T)$ for the smallest value of $k$ for which this is true, and call the integer $\alpha(T)$, the ascent of $T$. If no such integer exists, we shall say that $T$ has infinite ascent. In a similar way, $\left\{R\left(T^{h}\right)\right\}$ forms a descending sequence; the smallest integer for which $R\left(T^{k}\right)=R\left(T^{k+1}\right)$ is called the descent of $T$ and is denoted by $\delta(T)$. If no such integer exists, we shall say that $T$ has infinite descent.

The quantities $\alpha(T)$ and $\delta(T)$ were first discussed by F. Riesz [4] in his original investigation of compact linear operators. A comprehensive treatment of the properties of $\alpha(T)$ and $\delta(T)$ can be found in [6] pp. 271-284. The purpose of the present work is the consideration of the functions $\alpha(\lambda I-T)$ and $\delta(\lambda I-T)$ for complex $\lambda$. When no confusion can arise, we shall write these quantities as $\alpha(\lambda)$ and $\delta(\lambda)$ respectively.

DEFinition. Let $\Re_{T}$ denote the set $\{\lambda: \alpha(\lambda)$ and $\delta(\lambda)$ are finite $\}$. We shall refer to $\Re_{T}$ as the Riesz region of $T$.

If we write $n(\lambda)$ for the dimension of $N(\lambda I-T)$, i.e., the nullity of $\lambda I-T$ and $d(\lambda)$ for the codimension of $R(\lambda I-T)$, i.e., 
the defect of $\lambda I-T$, then it is customary to refer to the set $\{\lambda$ : $n(\lambda)$ and $d(\lambda)$ are finite $\}$ as the Fredholm region of $T$. We shall denote this region by $\widetilde{F}_{T}$. It should be observed that the above is a departure from traditional notation where $\alpha$ and $\beta$ are used for nullity and defect, respectively.

2. Remarks. From this point onwards, we shall assume that all operators are closed, with range and domain in $X$ unless otherwise stated.

1. It is well known that $\widetilde{\mho}_{T^{\prime}}$ is an open set and that $n(\lambda)-d(\lambda)$ is constant on each component of $\widetilde{\mho}_{r}$. These facts and a great many others are proven in papers by Gohberg and Krein [2] and by T. Kato [3]. We shall show below that $\mathfrak{F}_{T} \cap \Re_{T^{\prime}}$ is always open and that $\Re_{T}$ is open when certain other conditions are fulfilled. However the quantity $\delta(\lambda)-\alpha(\lambda)$ need not be constant on the components of $\Re_{T^{\prime}}$; for consider operator $T$ where $D(T) \neq X ; D(T) \neq\{0\}$ and $T x=x$ for $x \in D(T)$. Then $\Re_{T}$ is the entire complex plane $C$ but $\delta(\lambda)=1$, $\alpha(\lambda)=0$, when $\lambda \neq 1 ; \delta(1)=\alpha(1)=1$. However, if $D(T)=X$, then $\alpha(\lambda)=\delta(\lambda)$ on $\Re_{T}$ even in the absence of any topology in $X$. Proof of this fact can be found in [6] Theorem 5.41-E. Another notable difference between $\Re_{T^{\prime}}$ and $\widetilde{\mho}_{T}$ is seen from the theorem proven in [2]: if $B(X)$ denotes the space of bounded linear operators defined on $X$ and $\widetilde{F}_{r^{\prime}}=C$, then $X$ is finite dimensional. It is clear that no such restriction applies to $\Re_{T}$; indeed $\Re_{I}=C$.

2. If we adopt the usual notation of $\rho(T), P \sigma(T), C \sigma(T)$ and $R \sigma(T)$ for the resolvent set, point spectrum, continuous spectrum and residual spectrum respectively as given in [6], then it is known that for $T \in B(X), \delta(\lambda)=\infty$ if $\lambda \in C \sigma(T) \cup R \sigma(T)$. This is proven in [1]. Hence $\Re_{T}$ consists of $\rho(T)$ and possibly some elements of the point spectrum.

\section{Some preliminary lemmas.}

LEMMa 1. For any non negative integer $k$

(i) $n\left(T^{k}\right) \leqq \alpha(T) n(T)$

(ii) $d\left(T^{k}\right) \leqq \delta(T) d(T)$.

Proof. (i) We firstly observe that $\alpha(T)=0$ if and only if $n(T)=0$. Hence the product $\alpha(T) n(T)$ is well defined. We need only consider the case where both $\alpha(T)$ and $n(T)$ are finite. Let $\alpha(T)=p$. Then $n\left(T^{k}\right) \leqq n\left(T^{p}\right)$ for any $k$ and if we show $n\left(T^{k}\right) \leqq k n(T)$ for every nonnegative integer $k$, the result will follow. We proceed by induc- 
tion; clearly for $k=1, n\left(T^{k}\right) \leqq k n(T)$. Suppose we have shown its validity for $1 \leqq k \leqq s$. Then we can complete the proof by showing

$$
n\left(T^{s+1}\right)-n\left(T^{s}\right) \leqq n(T) .
$$

Let $N\left(T^{s+1}\right)=N\left(T^{s}\right) \oplus Y$. Choose $x_{1}, x_{2}, \cdots, x_{r}$ linearly independent in $Y$. Then these elements lie in $N\left(T^{s+1}\right)$ so that $T^{s} x_{i}(i=1,2, \cdots, r)$ lie in $N(T)$. But $\sum_{i=1}^{r} c_{i} T^{s} x_{i}=0$ implies $T^{s} \sum_{i=1}^{r}\left(c_{i} x_{i}\right)=0$ which would mean that $\sum_{i=1}^{r} c_{i} x_{i} \in N\left(T^{s}\right) \cap Y$. Therefore all $c_{i}$ must be zero. Hence the elements $\left\{T^{s} x_{i}: i=1,2, \cdots, r\right\}$ are linearly independent in $N(T)$. This implies the validity of (1) and completes the proof.

(ii) Again, since $\delta(T)$ is zero if and only if $d(T)$ is zero, the product $\delta(T) d(T)$ is well defined and we need only consider the case when $\delta(T)$ and $d(T)$ are finite. Again it suffices to prove that for each positive integer $k$,

$$
d\left(T^{k}\right) \leqq k d(T)
$$

Clearly (2) is valid for $k=1$; suppose we have shown its validity for $1 \leqq k \leqq s$. Let $R\left(T^{s+1}\right) \oplus Y=R\left(T^{s}\right)$ and take $y_{1}, y_{2}, \cdots, y_{r}$ linearly independent in $Y$. Then these element belong to $R\left(T^{s}\right)$ so that there exist $x_{1}, x_{2}, \cdots, x_{r}$ in $D\left(T^{s}\right)$ such that $y_{i}=T^{s} x_{i}, \quad i=1$, $2, \cdots, r$.

Suppose now we write $X=R(T) \oplus Z$ so that we can write $x_{i}=T x_{i}^{\prime}+z_{i}$ for some $x_{i}^{\prime} \in D(T)$ and $z_{i} \in Z, i=1,2, \cdots, r$. Then $\left\{z_{i}\right\}$ is a linearly independent set; for if $\sum_{i=1}^{r} c_{i} z_{i}=0$ then $\sum_{i=1}^{r} c_{i} T^{s} z_{i}=0$ so that $\sum_{i=1}^{r} c_{i} T^{s} x_{1}=\sum_{i=1}^{r} c_{i} T^{s+1} x_{i}^{\prime}$ i. e.,

$$
\sum_{i=1}^{r} c_{i} y_{i}=\sum_{i=1}^{r} c_{i} T^{s+1} x_{i}^{\prime} .
$$

But the left side of (3) lies in $Y$, the right side in $R\left(T^{s+1}\right)$. Hence $\sum_{i=1}^{r} c_{i} y_{i}=0$. Hence each $c_{i}$ is zero. This means that $\operatorname{dim} Y \leqq \operatorname{dim} Z$ so that

$$
d\left(T^{s+1}\right)-d\left(T^{s}\right) \leqq d(T)
$$

and hence (2) is valid for $k=s+1$. This completes the proof of (ii).

Lemma 2. If $\lambda \in \mathfrak{R}_{T^{\prime}} \cap \widetilde{F}_{T^{\prime}}$ and $T$ is densely defined, then $n(\lambda)=d(\lambda)$ and $\alpha(\lambda)=\delta(\lambda)$.

Proof. Without loss of generality, assume $\lambda=0$. Then, writing $\kappa(A)=d(A)-n(A)$ for any operator $A$, we can use Theorem 2.1 of 
[2] to write

$$
\kappa(A B)=\kappa(A)+\kappa(B)
$$

where $A, B$ are operators in $X$ with finite nullities and defects. As remarked at the end of the proof of the theorem cited, (4) is valid in all cases where $A, B$ act from one Banach space to another, the product $A B$ has a sense, and $A$ is densely defined. Moreover $A B$ has finite nullity and defect. In our case, we can write

$$
\kappa\left(T^{p}\right)=p \kappa(T)
$$

by induction from (4), for any positive integer $p$. Hence setting $p=k, k+1$ and subtracting we get

$$
\left[n\left(T^{k+1}\right)-n\left(T^{k}\right)\right]-\left[d\left(T^{k+1}\right)-d\left(T^{k}\right)\right]=n(T)-d(T) .
$$

On account of Lemma 1 , all quantities involved are finite. Choose $k$ greater than $\alpha(T)$ and $\delta(T)$; then left side of (6) reduces to zero and hence $n(T)=d(T)$. Finally, we can write

$$
n\left(T^{k+1}\right)-n\left(T^{k}\right)=d\left(T^{k+1}\right)-d\left(T^{k}\right)
$$

which makes it clear that $\alpha(T)=\delta(T)$.

4. Definitions. Suppose that the norm in $X$ is denoted by $\|\cdot\|$ and that we introduce a new norm into $D(T)$ by setting $|x|=\|x\|+$ $\|T x\|$. Then, as first shown in [5], $D(T)$ is closed with respect to $|\cdot|$ and can therefore be regarded as a Banach space. $T$ is then a closed operator defined on all of a Banach space so that, by the closed graph theorem, $T$ is bounded i. e., there exists $k$ such that $\|T x\| \leqq k|x|$ for each $x \in D(T)$. We shall write $|T|$ to denote the infimum of such $k$. If $S$ is another closed operator with $D(S) \supseteqq D(T)$, then the restriction of $S$ to $D(T)$ can also be regarded as a bounded operator with bound denoted by $|S|$.

Following [3], we define a quantity $\gamma(T)$ as the supremum of all $\lambda$ which satisfy $\lambda d(x, N(T)) \leqq\|T x\|$ for all $x \in D(T)$.

5. Consideration of $\Re_{T} \cap \mathfrak{F}_{T}$. Let $\lambda_{0}$ be a point in $\Re_{T^{\prime}} \cap \mathfrak{F}_{T^{\prime}}$; without loss of generality, we may assume $\lambda_{0}=0$. We define the following positive number:

$$
R_{p}=\left\{\begin{array}{cl}
\gamma(T) & \text { if } p=1 \\
2\left|\sin \frac{\Pi}{p}\right| \gamma(T) & \text { if } p>1 .
\end{array}\right.
$$

For each $p$, we know from [3], Lemma 341, that $T^{p}$ is a closed 
operator so that we can make $D\left(T^{p}\right)$ into a Banach space $X_{p}$ by introducing the norm $|x|_{(p)}=\|x\|+\left\|T^{p} x\right\|$. Then for $i=0,1, \cdots$, $p$, we can consider the restrictions of $T^{i}$ to $X_{p}$. Such restrictions being obviously closed operators, it follows from the closed graph theorem that they are bounded as operators from $X_{p}$ to $X$. Write $\left|T^{i}\right|_{(p)}$ to denote the respective bounds of these operators.

Define

$$
r_{p}=\left[1+\frac{\gamma\left(T^{p}\right)}{\left[1+\gamma\left(T^{p}\right)\right] \max _{0 \leqq i \leqq p-1}\left|T^{i}\right|_{(p)}}\right]^{1 / p}-1 .
$$

Finally, if $\alpha_{0}=\alpha(T), n_{0}=n(T), \delta_{0}=\delta(T)$ write

$$
\Gamma=\min _{1 \leqq p \leqq \alpha_{0} n_{0}+\delta_{0}+1} \min \left(r_{p}, R_{p}\right) \text {. }
$$

THEOREM 1. $\mathfrak{R}_{T} \cap \mathfrak{F}_{T^{\prime}}$ is an open set; indeed, if we take $\lambda=0$ as a point of $\Re_{T} \cap \widetilde{\mho}_{T}$, then the interior of the circle $|\lambda|=\Gamma$ lies in $\Re_{T^{\prime}} \cap \mathfrak{F}_{T^{*}}$.

Proof. By [3] Theorem 1, inside the circle $|\lambda|=\gamma(T), T-\lambda$ is a closed linear operator, $n(T-\lambda) \leqq n(T)$ and $R(T-\lambda)$ is closed. Moreover, we claim that inside the circle $|\lambda|=R_{p},(T-\lambda)^{p}-T^{p}$ is a closed operator.

$$
\text { For } \quad(T-\lambda)^{p}-T^{p}=\prod_{K=0}^{p-1}\left[T-\lambda-\left(\exp \frac{2 \pi K i}{p}\right) T\right]
$$

if $p>1$, and if we write $T_{K}=T\left(1-\exp \frac{2 \pi K i}{p}\right)$, the $T_{K}$ is a closed operator with finite nullity. Also

$$
\begin{aligned}
\gamma\left(T_{K}\right)=\inf _{x \notin N\left(T_{K}\right)} \frac{\left\|T_{K} x\right\|}{d\left(x, N\left(T_{K}\right)\right)} & =\left|1-\exp \frac{2 \pi K i}{p}\right| \inf _{x \notin N(T)} \frac{\|T x\|}{d(x, N(T))} \\
& \geqq 2\left|\sin \frac{\pi}{p}\right| \gamma(T)=R_{p} .
\end{aligned}
$$

Hence, if $|\lambda|<R_{p}$, then each factor in (8) is a closed linear operator with finite nullity so that by [3] Lemma 341, $(T-\lambda)^{p}-T^{p}$ is closed in this circle. Since the domain of this operator is $D\left(T^{p}\right)$, we can write

$$
\begin{aligned}
\left|(T-\lambda)^{p}-T^{p}\right|_{(p)} & \leqq \sum_{i=0}^{p-1}\left(\begin{array}{c}
p \\
i
\end{array}\right)\left|T^{i}\right|_{(p)}|\lambda|^{p-i} \\
& \leqq\left[(1+|\lambda|)^{p}-1\right] \max _{0 \leqq i \leqq p-1}\left|T^{i}\right|_{(p)}
\end{aligned}
$$


If $|\lambda|<r_{p}$, this shows that $\left|(T-\lambda)^{p}-T^{p}\right|_{(p)} \leqq \frac{\gamma\left(T^{p}\right)}{1+\gamma\left(T^{p}\right)}$.

By [3], Theorem 1a, if $|\lambda|<\min \left(r_{p}, R_{p}\right)$, then

$$
\left.\begin{array}{l}
n\left[(T-\lambda)^{p}\right] \leqq n\left(T^{p}\right) \\
d\left[(T-\lambda)^{p}\right] \leqq d\left(T^{p}\right) \\
\kappa\left[(T-\lambda)^{p}\right]=\kappa\left(T^{p}\right)
\end{array}\right\}
$$

for $p>1$.

Observe that (9) also holds for $p=1$; for we can apply [3] Theorem 1 directly to $T$ and $-\lambda I$.

Now, if $|\lambda|<\Gamma$,

$$
\begin{aligned}
n\left[(T-\lambda)^{p}\right. & \leqq n\left(T^{p}\right) & & 1 \leqq p \leqq \alpha_{0} n_{0}+1 \\
& \leqq \alpha_{0} n_{0} & & \text { by Lemma } 1 .
\end{aligned}
$$

Hence $n\left[(T-\lambda)^{p}\right]$ cannot be strictly increasing for $1 \leqq p \leqq \alpha_{0} n_{0}+1$; thus $\alpha(\lambda) \leqq \alpha_{0} n_{0}$.

Finally, from (9), we can write

$$
\begin{aligned}
& n\left[(T-\lambda)^{K}\right]-d\left[(T-\lambda)^{K}\right]=n\left(T^{K}\right)-d(T)^{K} \\
& n\left[(T-\lambda)^{K+1}\right]-d\left[(T-\lambda)^{K+1}\right]=n\left(T^{K+1}\right)-d\left(T^{K+1}\right)
\end{aligned}
$$

with $K=\alpha_{0} n_{0}+\delta_{0}$. Now $\alpha_{0} n_{0}+\delta_{0}$ exceeds both $\alpha_{0}$ and $\delta_{0}$ and since all quantities involved in the above equalities are finite by Lemma 1 , we get

$$
d\left[(T-\lambda)^{K+1}\right]=d\left[(T-\lambda)^{K}\right]
$$

i. e., $\delta(\lambda) \leqq \alpha_{0} n_{0}+\delta_{0}$ in the circle $|\lambda|<\Gamma$.

LEMMA 3. (This is essentially [2], Lemma 3.1 in a slightly more general setting.)

Let $T$ be an operator with $0 \in \mathfrak{F}_{n^{*}}$ and let $S$ be an operator with $D(S) \supseteqq D(T)$. Then if $|S|$ is defined by the norm $\|x\|+\|T x\|$ on $D(T)$, there exists $\varepsilon>0$ such that $n(T+S)$ is constant for $0<|S|<\varepsilon$.

Proof. The original formulation of this Lemma considers $A, B$ operators with domains in Banach space $B_{1}$ and ranges in Banach space $B_{2} ; 0 \in \mathfrak{F}_{A}$ and $B$ is a bounded linear operator. The conclusion is that there exists $\varepsilon>0$ such that $n(A-\lambda B)$ is constant for $0<|\lambda|<\varepsilon$.

In our case, take $B_{1}$ to be $D(T)$ with the norm $|x|=\|x\|+\|T x\|$ and $B_{2}=X, A=T$. If $S$ is the restriction of $S$ to $B_{1}$, so that $S$ is a bounded operator, take $B=-S /|S|$. Then we can conclude that 
there exists $\varepsilon>0$ such that $n(T+\lambda S /|S|)$ is constant for $0<|\lambda|<\varepsilon$. In particular, if $0<|S|<\varepsilon$, then $n(T+S)$ is constant.

THEOREM 2. Let $\Omega$ be a component of $\Re_{T^{\prime}} \cap \widetilde{\mho}_{T^{\prime}}$ where $T$ is densely defined. Then $\alpha(\lambda)$ and $\delta(\lambda)$ will be equal on $\Omega$ (by Lemma 2 ) and the common value is constant except at isolated points.

Proof. Let $K$ be a positive integer. Then by Lemma 1, $n\left[(T-\lambda)^{K}\right]$ is finite in $\Omega$. Let $n_{K}=\min _{\Omega} n\left[(T-\lambda)^{K}\right]$ and suppose $n\left[\left(T-\lambda_{0}\right)^{K}\right]=n_{K}$ and $n\left[\left(T-\lambda_{1}\right)^{K}\right]>n_{K}$. Join $\lambda_{1}$ to $\lambda_{0}$ by a curve $\Gamma_{K}$ lying in $\Omega$. We now apply Lemma 3 to the operators $A=(T-\lambda)^{K}$ $B=(T-\mu-\lambda)^{K}-(T-\lambda)^{K}$ for any point $\lambda$ on $\Gamma_{K}$. Then $n\left[(T-\mu-\lambda)^{K}\right]$ is constant for $0<|B|<\varepsilon$ and since $|B|$ is a continuous function of $\mu$, we get a deleted neighbourhood of $\lambda$ in which $n\left[(T-\mu)^{K}\right]$ is constant. The compactness of $\Gamma_{K}$ enables us to deduce in the usual way that there exists an open set $U_{K}$ containing $\Gamma_{K}$ such that $n\left[(T-\lambda)^{K}\right]$ is constant for $\lambda \in U_{K}$ except at a finite number of points. In particular, relations (9) imply that in some neighbourhood of $\lambda_{0}, n\left[(T-\lambda)^{K}\right]$ takes a constant value $n_{K}$. Hence in $U_{K}, n\left[(T-\lambda)^{K}\right]=n_{K}$ except at a finite number of points. In particular, in some deleted neighbourhood of $\lambda_{1}, \quad n\left[(T-\lambda)^{K}\right]=n_{K}$. Thus on $\Omega, \quad n\left[(T-\lambda)^{K}\right]=n_{K}$ except at isolated points. Let the set of exceptional points be denoted $\Omega_{K}$. Choose $\lambda^{*}$ with the property that $\lambda^{*} \notin \Omega_{K}$ for all $K$. This can be done simply by taking any line segment $l$ in $\Omega$ and choosing $\lambda^{*}$ to be any points of $l-\bigcup_{1}^{\infty} \Omega_{K}$. Let $\alpha\left(\lambda^{*}\right)=\alpha^{*}$ and $\delta\left(\lambda^{*}\right)=\delta^{*}$. By Lemma 2, $\alpha^{*}=\delta^{*}$. Consider $\lambda \in \Omega-\bigcup_{1}^{1+\alpha^{*}} \Omega_{K}$. Then $n\left[(T-\lambda)^{K}\right]=n\left[\left(T-\lambda^{*}\right)^{K}\right]$ for each $k, 1 \leqq k \leqq 1+\alpha^{*}$. Hence $\alpha(\lambda)=\alpha^{*}$ and by Lemma $2, \delta(\lambda)=\delta^{*}$ for $\lambda \in \Omega-\bigcup_{1}^{1+\alpha *} \Omega_{K}$.

Corollary. If $\Omega \cap \rho(T) \neq \varnothing$, then $\Omega \cap \sigma(T)$ consists of poles of the resolvent $R_{\lambda}(T)$.

Proof. Since $\rho(T)$ is an open set in which $\alpha(\lambda)=\delta(\lambda)=0, \alpha(\lambda)$ and $\delta(\lambda)$ must be zero on $\Omega$ except at isolated points. It is known that such a point $\lambda_{0}$ is a pole of $R_{\lambda}(T)$ if $R\left[\left(T-\lambda_{0}\right)^{\alpha\left(\lambda_{0}\right)}\right]$ is closed. But $\left(T-\lambda_{0}\right)^{\alpha\left(\lambda_{0}\right)}$ has finite codimension by Lemma 1 and hence, by [3] Lemma 332 , closed range.

\section{Consideration of $\mathfrak{R}_{T}$.}

THEOREM 3. Let $T$ be a closed linear operator such that $\alpha(T)=$ $p<\infty$. Suppose that there exists $\varepsilon>0$ such that if $|\lambda|<\varepsilon$, then it is possible to write 


$$
X=N\left[(T-\lambda)^{p}\right] \oplus S(\lambda)
$$

in such a manner that

$$
S(\lambda) \cap D\left(T^{p+1}\right)=S(0) \cap D\left(T^{p+1}\right) .
$$

Then if $R\left(T^{p+1}\right)$ is closed, there exists $\rho>0$ such that $\alpha(\lambda) \leqq \alpha(T)$ for $|\lambda|<\rho$.

Proof. Write $S(0)=S$ and define $D=S \cap D\left(T^{p+1}\right)$. Let $T_{p}$ be the restriction of $T^{p+1}$ to $D$. We first show that

$$
N\left(T^{p+1}\right)=N\left(T^{p}\right) \oplus N\left(T_{p}\right) .
$$

Suppose $x \in N\left(T^{p}\right) \cap N\left(T_{p}\right)$; then

$$
x \in N\left(T^{p}\right) \cap D\left(T_{p}\right)=N\left(T^{p}\right) \cap S \cap D\left(T^{p+1}\right)=\{0\}
$$

by (10). Hence $N\left(T^{p}\right) \oplus N\left(T_{p}\right)$ is well defined. Now let $x \in N\left(T^{p+1}\right)$. By (10), we can write $x=x_{1}+x_{2}$ with $x_{1} \in N\left(T^{p}\right)$ and $x_{2} \in S$. Now $x_{2}=x-x_{1} \in N\left(T^{p+1}\right) \cap S \subseteq D$, and $T_{p} x_{2}=T^{p+1} x_{2}=0$. Hence $N\left(T^{p+1}\right)=$ $N\left(T^{p}\right) \oplus N\left(T_{p}\right)$.

We next verify that $R\left(T_{p}\right)=R\left(T^{p+1}\right)$. It is obvious that $R\left(T_{p}\right) \subseteq R\left(T^{p+1}\right)$. Suppose then that $x \in R\left(T^{p+1}\right)$; then $x=T^{p+1} y$ for some $y \in D\left(T^{p+1}\right)$. Use (10) again to write $y=y_{1}+y_{2}$ with $y_{1} \in N\left(T^{p}\right)$, $y_{2} \in S$. Then $T^{p+1} y=T^{p+1} y_{2}$ and since $y_{2} \in S \cap D\left(T^{p+1}\right)$, we have $x=T^{p+1} y_{2}=T_{p} y_{2}$. Hence $R\left(T_{p}\right)=R\left(T^{p+1}\right)$.

If we now repeat the same arguments replacing $T$ by $T-\lambda$ we obtain an operator $T_{p}(\lambda)$ with domain $S(\lambda) \cap D[(T-\lambda)$, range equal to $R\left[(T-\lambda)^{p+1}\right]$ such that

$$
N\left[(T-\lambda)^{p+1}\right]=N\left[(T-\lambda)^{p}\right] \oplus N\left[T_{p}(\lambda)\right] .
$$

Now by assumption, $N\left(T_{p}\right)=\{0\}$ and $T_{p}$ has closed range. Hence $T_{p}^{-1}$ can be considered as a bounded linear operator on $R\left(T_{p}\right)$; hence there exists $m>0$ such that $\left\|T_{p} x\right\| \geqq m|x|$ for all $x \in D\left(T_{p}\right)$ where $|x|$ is defined, as in $\S 4$, by $|x|=\|x\|+\left\|T_{p} x\right\|$. For $|\lambda|<\varepsilon$, $D\left[T_{p}(\lambda)\right]=D\left(T_{p}\right)$ so that $T_{p}(\lambda)-T_{p}$ is defined on $D\left(T_{p}\right)$ and has bound $\left|T_{p}(\lambda)-T_{p}\right|$ where

$$
\begin{aligned}
\left|T_{p}(\lambda)-T_{p}\right| & =\sup \left\{\frac{\|\left(T_{p}(\lambda)-T_{p}\right) x||}{\|x\|+\left\|T_{p} x\right\|}: x \in D\left(T_{p}\right), x \neq 0\right\} \\
& \leqq \sup \left\{\frac{\left\|\left[(T-\lambda)^{p+1}-T^{p+1}\right] x\right\|}{\|x\|+\left\|T^{p+1} x\right\|}: x \in D\left(T^{p+1}\right) x \neq 0\right\} \\
& =\left|(T-\lambda)^{p+1}-T^{p+1}\right|_{(p+1)} \text { where }|\cdot|_{(p+1)} \text { is defined in } \\
& \text { the proof of Theorem } 1 \\
& \leqq\left(|T|_{(p+1)}+|\lambda|\right)^{p+1}-|T|_{\substack{p+1) \\
(p+1)}}
\end{aligned}
$$


Let $\lambda$ be chosen such that $|\lambda|<\varepsilon$ and $\left(|T|_{(p+1)}+|\lambda|\right)^{p+1}-|T|_{(p+1)}^{p+1}<$ $m / 3$. Then

$$
\begin{aligned}
\left\|T_{p}(\lambda) x\right\| & =\left\|T_{p} x+\left[T_{p}(\lambda)-T_{p}\right] x\right\| \geqq\left\|T_{p} x\right\|-\left\|\left[T_{p}(\lambda)-T_{p}\right] x\right\| \\
& \geqq m|x|-\frac{m}{3}|x|=\frac{2 m}{3}|x| \text { for } x \in D\left[T_{p}(\lambda)\right] .
\end{aligned}
$$

Hence $N\left[T_{p}(\lambda)\right]=\{0\}$ so that $\alpha(\lambda) \leqq \alpha(T)$ if $|\lambda|$ is suitably chosen; in fact, if $|\lambda|<\varepsilon$ and $|\lambda|<\left[|T|_{(p+1)}^{p+1}+m / 3\right]^{1 /(p+1)}-|T|_{(p+1)}$. This concludes the proof.

6.1 We shall assume from now on that $T$ and all its iterates are densely defined. Then $T$ has an adjoint $T^{*}$ defined in the space $X^{*}$ of bounded linear functionals on $X$. We shall write $\left\langle x, x^{*}\right\rangle$ to denote the value of functional $x^{*}$ at $x$.

Definition. Operator $A$ is said to be an extension of operator $B$ if $D(A) \supseteqq D(B)$ and $A x=B x$ for $x \in D(B)$. If $D(A)$ can be written as $D(A)=D(B) \oplus Y$ where $Y$ is a subspace of dimension $k$, then we call $A$ a $k$-dimensional extension of $B$ and write $[A: B]=k$.

Lemma 4. $\left(T^{K}\right)^{*}$ is an extension of $\left(T^{*}\right)^{K}$ for any positive integer $K$.

Proof. The lemma is trivial for $K=1$; suppose it has been verified for $K \leqq p$. Let $x^{*} \in D\left[\left(T^{*}\right)^{p+1}\right]$. Then $x^{*} \in D\left[\left(T^{*}\right)^{p}\right]$ and $\left(T^{*}\right)^{p} x^{*} \in D\left(T^{*}\right)$. Hence for any $x \in D\left(T^{p+1}\right)$, we can write

$$
\begin{aligned}
\left\langle T^{p+1} x, x^{*}\right\rangle & =\left\langle T x,\left(T^{p}\right)^{*} x^{*}\right\rangle \\
& =\left\langle T x,\left(T^{*}\right)^{p} x^{*}\right\rangle \text { by assumption } \\
& =\left\langle x,\left(T^{*}\right)^{p+1} x^{*}\right\rangle .
\end{aligned}
$$

Hence $x^{*} \in D\left[\left(T^{p+1}\right)^{*}\right]$ and $\left(T^{*}\right)^{p+1} x^{*}=\left(T^{p+1}\right)^{*}$. This completes the proof.

Definition. We shall say that $T$ is of finite type if, for each $K$, $\left(T^{K}\right)^{*}$ is a finite dimensional extension of $\left(T^{*}\right)^{K}$. If, in addition, $\left[\left(T^{K}\right)^{*}:\left(T^{*}\right)^{K}\right]$ is a bounded sequence, we shall say that $T$ is of bounded type.

Example. Every $T \in B(X)$ is of bounded type since $\left(T^{K}\right)^{*}=\left(T^{*}\right)^{K}$ for all $K$.

LEMma 5. Suppose that $T$ is of finite type and that $R\left(T^{K}\right)$ is closed for each positive integer $K$. Then 
(a) $\alpha\left(T^{*}\right)$ is finite if $\delta(T)$ is finite

(b) $\alpha(T)$ is finite if $\delta\left(T^{*}\right)$ is finite.

If, in addition, $T$ is of bounded type, then we also have

(c) $\delta(T)$ is finite if $\alpha\left(T^{*}\right)$ is finite

(d) $\delta\left(T^{*}\right)$ is finite if $\alpha(T)$ is finite.

Proof. By [4], Lemma 335, since $T$ is a closed operator with closed range

$$
\left.\begin{array}{l}
N\left(T^{*}\right)=R(T)^{\perp} \\
R\left(T^{*}\right)=N(T)^{\perp}
\end{array}\right\}
$$

where for any $Y \subseteq X, Y^{\perp}=\left\{x^{*} \in X^{*}:\left\langle y, x^{*}\right\rangle=0 \forall y \in Y\right\}$.

For each positive integer $K$, we can write, by assumption

$$
\left[R\left(T^{K}\right)\right]^{\perp}=N\left[\left(T^{K}\right)^{*}\right]=N\left[\left(T^{*}\right)^{K}\right] \oplus Y_{K}
$$

where clearly $Y_{K}$ must be of finite dimension. Now for $K>\delta(T)$, it is clear from (13) that $N\left[\left(T^{*}\right)^{K}\right] \oplus Y_{K}$ must be independent of $K$. But if $\alpha\left(T^{*}\right)$ is infinite, $\left.\left\{N\left[T^{*}\right)^{K}\right]\right\}$ is a strictly increasing sequence of subspaces so that $\left\{Y_{K}\right\}$ would need to be strictly decreasing. This is not possible for finite dimensional subspaces. Hence $(a)$ is verified. Conversely, if $\alpha\left(T^{*}\right)$ is finite, then $\delta(T)$ must also be finite when $T$ is of bounded type. For were $\delta(T)$ infinite, $\left\{\left[R\left(T^{K}\right)\right]^{\perp}\right\}$ would be strictly increasing and for $K>\alpha\left(T^{*}\right),\left\{N\left[\left(T^{*}\right)^{K}\right]\right\}$ is independent of $K$. By (13), this would imply that $\left\{Y_{K}\right\}$ is strictly increasing. For $T$ of bounded type, this is not possible. This proves $(c)$.

Next, we write, for each nonnegative integer $K$,

$$
R\left[\left(T^{K}\right)^{*}\right]=R\left[\left(T^{*}\right)^{K}\right] \oplus Z_{K}
$$

and again we can deduce from our assumptions that each $Z_{K}$ is finite dimensional. But, from (12),

$$
\begin{aligned}
R\left[\left(T^{K}\right)^{*}\right] & =\left[N\left(T^{K}\right)\right]^{\perp} \\
& \cong\left[X / N\left(T^{K}\right)\right]^{*} \text { by } \quad[6] \text { p. } 227,
\end{aligned}
$$

where $\cong$ indicates linear homeomorphism.

Now suppose $X=N\left(T^{K}\right) \oplus W_{K}$. Then $W_{K}$ is isomorphic to $X / N\left(T^{K}\right)$.

Using $\equiv$ to denote isomorphism, we obtain

$$
\begin{aligned}
R\left[\left(T^{K}\right)^{*}\right] & \equiv W_{K}^{*} \\
& \equiv X^{*} / W_{K}^{\perp} \quad \text { by [2], p. } 188 .
\end{aligned}
$$

Let $\alpha(T)$ be infinite; then $\left\{W_{K}\right\}$ is strictly descending; $\left\{W_{K}^{\perp}\right\}$ strictly ascending. By (16), $\left\{R\left[\left(T^{K}\right)^{*}\right]\right\}$ is strictly descending. Now, if $\delta\left(T^{*}\right)$ 
is finite, then by (14), $\left\{Z_{K}\right\}$ must be strictly descending. But this is not possible. Hence (b) is proved.

Finally, suppose $\delta\left(T^{*}\right)$ infinite and $\alpha(T)$ is finite. Then $\left\{W_{K}\right\}$ is independent of $K$ for $K>\alpha(T)$. From (16) and (14), we deduce that $\left\{Z_{K}\right\}$ must be strictly increasing, contrary to assumption. This verifies (d) and completes the proof.

THEOREM 4. Suppose $T$ is a closed linear operator such that $\delta(T)=q<\infty$. Let $T$ be of bounded type. Then $\alpha\left(T^{*}\right)<\infty$. Suppose that $T^{*}$ satisfies the assumptions of Theorem 3 and that there exists $\eta>0$ such that $(T-\lambda)^{*}$ is of bounded type for $|\lambda|<\eta$. Then there exists $\sigma>0$ such that $\delta(\lambda)$ is finite in the circle $|\lambda|<\sigma$.

Proof. The assertion that $\alpha\left(T^{*}\right)$ is finite follows directly from Lemma 5. Moreover since $R\left(T^{K}\right)$ is closed for $K=1+\alpha\left(T^{*}\right)$, then by [4] Lemma 324, $R\left[\left(T^{K}\right)^{*}\right.$ ] is closed for $K=1+\alpha\left(T^{*}\right)$. By assumption $\left(T^{K}\right)^{*}$ is a finite dimensional extension of $\left(T^{*}\right)^{K}$ so that by [3] Lemma 333, $\left(T^{*}\right)^{K}$ has closed range. We now apply Theorem 3 to $T^{*}$ and deduce that $T^{*}-\lambda$ has finite ascent for $|\lambda|<\rho^{*}$ for some $\rho^{*}>0$. Now $(T-\lambda)^{*}=T^{*}-\lambda$ so that by Lemma 5 , we can conclude that if $\sigma=\min \left(\rho^{*}, \eta\right)$, then $\delta(\lambda)$ is finite in the circle $|\lambda|<\sigma$. This concludes the proof.

In view of the additional hypothesis regarding the nature of $(T-\lambda)^{*}$, it is of some interest to examine the relationship between extensions and their adjoints. The following lemmas shed some light on the situation.

Lemma 6. Suppose $A_{1}$ is an extension of $A_{2}$ and $\left[A_{1}: A_{2}\right]=k$. Then $A_{2}^{*}$ is an extension of $A_{1}^{*}$ and if $\overline{D\left(A_{1}\right)}=\overline{D\left(A_{2}\right)}$, then $\left[A_{2}^{*}: A_{1}^{*}\right]=k$.

Proof. It is well known that $A_{2}^{*}$ is an extension of $A_{1}^{*}$ and this fact is trivial to verify. Let $\overline{D\left(A_{1}\right)}=\overline{D\left(A_{2}\right)}=X_{0}$ and define a mapping $E$

$$
E: \quad X^{*} \times X_{0}^{*} \rightarrow\left(X_{0} \times X\right)^{*}
$$

by means of

$$
E(f, \mathrm{~g})-(x, y) \rightarrow f(y) g(x) .
$$

If the usual norm topology is introduced into the Cartesian products, then we can show that $E$ established a linear homeomorphism between $X^{*} \times X_{0}^{*}$ and $\left(X_{0} \times X\right)^{*}$. It is easy to see that $E$ is a linear map; moreover $E$ is surjective, for if $F \in\left(X_{0} \times X\right)^{*}$, we have $g \in X_{0}^{*}$ defined by $x \rightarrow F(x, 0)$ and $f \in X^{*}$ defined by $y \rightarrow F(0, y)$ so that

$$
E(f, g):(x, y) \rightarrow f(y)+g(x)=F(x, y) .
$$


$E$ is also injective, for if $E(f, g)=0$, then $f(y)+g(x)=0$ for all $x \in X, y \in X_{0}$. This is possible if and only if $f=g=0$. Finally, we can see that $E$ is continuous; for

$$
|E(f, g)(x, y)| \leqq\|f\|\|y\|+\|g\|\|x\| \leqq(\|f\|+\|g\|)(\|x\|+\|y\|) \text {. }
$$

By the closed graph theorem, $E^{-1}$ is also continuous. Hence we have shown that $E$ is a linear homeomorphism.

We next observe that if we write $G(T)$ to denote the graph of $T$, then

$$
E\left\{G\left(A_{i}^{*}\right)\right\}=\left\{G\left(-A_{i}\right)\right\}^{\perp} \quad i=1,2
$$

where $\left\{G\left(-A_{i}\right)\right\}^{\perp}$ denotes the elements $F$ in $\left(X_{0} \times X\right)^{*}$ such that $F(x, y)=0$ for all $(x, y) \in G\left(-A_{i}\right)$.

For, if $x \in D\left(A_{i}\right)$ and $f \in D\left(A_{i}^{*}\right)$, then

$$
E\left(f, A_{i}^{*} f\right)\left(x,-A_{i} x\right)=A_{i}^{*} f(x)-f\left(A_{i} x\right)=0
$$

so that $E\left\{G\left(A_{i}^{*}\right)\right\} \subseteq\left\{G\left(-A_{i}\right)\right\}^{\perp}$.

On the other hand, if $E(f, g) \in\left\{G\left(-A_{i}\right)\right\}^{\perp}$, then $E(f, g)\left(x,-A_{i} x\right)=$ 0 for all $x \in D\left(A_{i}\right)$. Then $f\left(A_{i} x\right)=g(x)$ for all $x \in D\left(A_{i}\right)$ so that $f \in D\left(A_{i}^{*}\right)$ and $g=T^{*} f$. Hence any $E(f, g)$ in $\left\{G\left(-A_{i}\right)\right\}^{\perp}$ is of the form $E\left(f, T^{*} f\right)$. This proves the validity of (17).

Now

$$
\begin{aligned}
E\left\{G\left(A_{i}^{*}\right)\right\} & =\left\{G\left(-A_{i}\right)\right\}^{\perp}=\left\{X_{0} \times X / G\left(-A_{i}\right)\right\}^{*} \text { by [6] p. } 227 \\
& \equiv\left\{\left(X_{0} \times X\right) \ominus G\left(-A_{i}\right)\right\}^{*} .
\end{aligned}
$$

Now suppose $\left(X_{0} \times X\right) \ominus G\left(-A_{i}\right)=X_{i}$. Then by [6] p. 188,

$$
X_{i}^{*}=\left(X_{0} \times X\right)^{*} / X_{i}^{\mathrm{L}}
$$

where $X_{i}^{\perp}=\left\{F: F \in\left(X_{0} \times X\right)^{*} ; F(x, y)=0\right.$ for all $\left.(x, y) \in X_{i}\right\}$.

It is easy to verify that $D\left(A_{i}\right)$ is isomorphic to $G\left(-A_{i}\right)$ by means of the natural mapping $x \rightarrow\left(x,-A_{i} x\right)$. Hence, $X_{2} \ominus X_{1}$ is a $k$ dimensional subspace and from (19), $X_{1}^{*} \ominus X_{2}^{*}$ is also $k$ dimensional. Finally from (18), we see that $E\left(G\left(A_{2}^{*}\right) \ominus G\left(A_{1}^{*}\right)\right)$ is $k$-dimensional from which we easily deduce that

$$
\left[A_{2}^{*}: A_{1}^{*}\right]=k \text {. }
$$

Lemma 7. Suppose $T$ is of finite, resp. bounded type and $\overline{D\left[\left(T^{K}\right)^{*}\right)}=\overline{D\left[\left(T^{*}\right)^{K}\right]}$ for each positive integer $K$. Moreover, let either of the following conditions hold:

(i) $\left[\left(T^{K}\right)^{* *}: T^{K}\right]$ is a sequence of finite terms, resp. bounded sequence

(ii) $X$ is reflexive.

Then $T^{*}$ is of finite, resp. bounded, type. 
Proof. To begin with, it is well known that if $X$ is reflexive, then $T^{* *}=T$ for any closed linear operator $T$. Hence condition (ii) implies condition (i). Suppose condition (i) holds. Then we have

$$
\left[\left(T^{K}\right)^{*}:\left(T^{*}\right)^{K}\right]=m_{K}<\infty
$$

and

$$
\left[\left(T^{K}\right)^{* *}: T^{K}\right]=n_{K}<\infty .
$$

By Lemma 6, (20) yields

$$
\left[\left(\left(T^{*}\right)^{K}\right)^{*}:\left(T^{K}\right)^{* *}\right]=m_{K}
$$

and this together with (21) gives

$$
\left[\left(\left(T^{*}\right)^{K}\right)^{*}: T^{K}\right]=m_{K}+n_{K} .
$$

But applying Lemma 4 to $T^{*}$ we get

$$
\left(\left(T^{*}\right)^{K}\right)^{*} \supseteqq\left(T^{* *}\right)^{K} \supseteqq T^{K}
$$

and from (22) and (23) we deduce

$$
\left[\left(\left(T^{*}\right)^{K}\right)^{*}:\left(T^{* *}\right)^{K}\right] \leqq m_{K}+n_{K} .
$$

But this gives exactly the required conclusion.

\section{BIBLIOGRAPHY}

1. S. R. Caradus, Operators of Riesz type (to be published).

2. I. C. Gohberg and M. G. Krein, The basic propositions on defect numbers, root numbers and indices of linear operators, Uspehi Mat. Nauk. (N. S.) 12 (1957) No. 2 (74), 43-118; A. M. S. Trans. Series 2, v. 13.

3. T. Kato, Perturbation theory for nullity, deficiency and other quantities of linear operators, Journ. d'Anal. Math. 6 (1958), 261-322.

4. F. Riesz, Uber lineare Funktionalgleichungen, Acta Math. 41 (1918), 71-98.

5. B. Sz.-Nagy, Perturbations des transformations lineaires fermees, Acta Sci. Math. Szeged 14 (1951), 125-137.

6. A. E. Taylor, Introduction to Functional Analysis, John Wiley and Sons, 1958.

Received August 5, 1965.

QUEEN'S UNIVERSITY

Kingston, Ontario, CANAda 



\section{PACIFIC JOURNAL OF MATHEMATICS}

\section{EDITORS}

\author{
H. SAMELSON, \\ Stanford University \\ Stanford, California \\ R. M. BLUMENTHAL \\ University of Washington \\ Seattle, Washington 98105
}

\author{
*J. DugundJI \\ University of Southern California \\ Los Angeles, California 90007 \\ RICHARD ARENS \\ University of California \\ Los Angeles, California 90024
}

\section{ASSOCIATE EDITORS}
E. F. BECKENBACH
B. H. NeUmanN
F. WOLF
K. YOSIDA

\section{SUPPORTING INSTITUTIONS}

\author{
UNIVERSITY OF BRITISH COLUMBIA \\ CALIFORNIA INSTITUTE OF TECHNOLOGY \\ UNIVERSITY OF CALIFORNIA \\ MONTANA STATE UNIVERSITY \\ UNIVERSITY OF NEVADA \\ NEW MEXICO STATE UNIVERSITY \\ OREGON STATE UNIVERSITY \\ UNIVERSITY OF OREGON \\ OSAKA UNIVERSITY \\ UNIVERSITY OF SOUTHERN CALIFORNIA
}

\author{
STANFORD UNIVERSITY \\ UNIVERSITY OF TOKYO \\ UNIVERSITY OF UTAH \\ WASHINGTON STATE UNIVERSITY \\ UNIVERSITY OF WASHINGTON \\ * * * \\ AMERICAN MATHEMATICAL SOCIETY \\ CHEVRON RESEARCH CORPORATION \\ TRW SYSTEMS \\ NAVAL ORDNANCE TEST STATION
}

Mathematical papers intended for publication in the Pacific Journal of Mathematics should be typewritten (double spaced). The first paragraph or two must be capable of being used separately as a synopsis of the entire paper. It should not contain references to the bibliography. Manuscripts may be sent to any one of the four editors. All other communications to the editors should be addressed to the managing editor, Richard Arens at the University of California, Los Angeles, California 90024 .

50 reprints per author of each article are furnished free of charge; additional copies may be obtained at cost in multiples of 50 .

The Pacific Journal of Mathematics is published monthly. Effective with Volume 16 the price per volume (3 numbers) is $\$ 8.00$; single issues, $\$ 3.00$. Special price for current issues to individual faculty members of supporting institutions and to individual members of the American Mathematical Society: $\$ 4.00$ per volume; single issues $\$ 1.50$. Back numbers are available.

Subscriptions, orders for back numbers, and changes of address should be sent to Pacific Journal of Mathematics, 103 Highland Boulevard, Berkeley 8, California.

Printed at Kokusai Bunken Insatsusha (International Academic Printing Co., Ltd.), No. 6, 2-chome, Fujimi-cho, Chiyoda-ku, Tokyo, Japan.

PUBLISHED BY PACIFIC JOURNAL OF MATHEMATICS, A NON-PROFIT CORPORATION

The Supporting Institutions listed above contribute to the cost of publication of this Journal, but they are not owners or publishers and have no responsibility for its content or policies.

* Paul A. White, Acting Editor until J. Dugundji returns. 


\section{Pacific Journal of Mathematics}

\section{Vol. 18, No. 3 \\ May, 1966}

William George Bade and Philip C. Curtis, Jr., Embedding theorems for commutative Banach algebras .......................... 391

Wilfred Eaton Barnes, On the $\Gamma$-rings of Nobusawa ................. 411

J. D. Brooks, Second order dissipative operators ................ 423

Selwyn Ross Caradus, Operators with finite ascent and descent ........ 437

Earl A. Coddington and Anton Zettl, Hermitian and anti-hermitian properties of Green's matrices .......................... 451

Robert Arnold Di Paola, On sets represented by the same formula in distinct consistent axiomatizable Rosser theories ................... 455

Mary Rodriguez Embry, Conditions implying normality in Hilbert space ...........................................

Garth Ian Gaudry, Quasimeasures and operators commuting with convolution ................................... 461

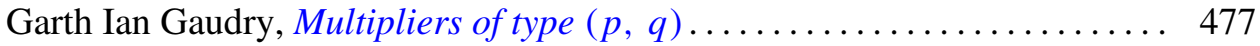

Ernest Lyle Griffin, Jr., Everywhere defined linear transformations affiliated with rings of operators .................................. 489

Philip Hartman, On the bounded slope condition ................ 495

David Wilson Henderson, Relative general position ................ 513

William Branham Jones, Duality and types of completeness in locally

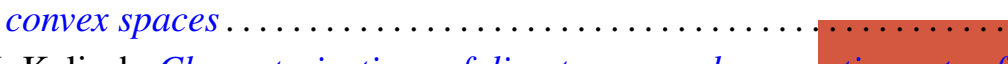

G. K. Kalisch, Characterizations of direct sums and commuting sets of Volterra operators....

Ottmar Loos, Über eine Beziehung zwischen Malcev-Algebren und Lietripelsystemen

Ronson Joseph Warne, A class of bisimple inverse semigroups . . . 